\title{
Presentation and outcome of twenty patients with synchronous stage IV rectal carcinoma
}

\author{
Mohamed A Aboziada ${ }^{1,2}$, Alia M Attia ${ }^{1}$, Abelaziz A Alhamad ${ }^{2}$ \\ ${ }^{I}$ Department of Radiation Oncology, South Egypt Cancer Institute, University of Assiut, Egypt. \\ ${ }^{2}$ Department of Oncology, Prince Sultan Military Medical City, Riyadh Saudi Arabia.
}

Received January 07, 2014; Revised April 28, 2014; Accepted May 04, 2014; Published Online June 15, 2014

\section{Original Article}

\begin{abstract}
Purpose: Palliative treatment techniques for advanced stage rectal cancer should be designed according to the patients' major symptoms. Combined chemo-radiation therapy is effective choice for symptomatic patients with good performance status. In this study, we reviewed our patients' stage IV rectal carcinoma in regard to most common presentation, outcome and possible prognostic features. Methods Medical chart of twenty patients who were diagnosed with stage IV rectal carcinoma, were reviewed based on the hospital database information, which included images, radiotherapy charts, and their follow up notes. Results: All patients were young with age less than 40 years. Bleeding per rectum, pain, and symptoms of obstruction were the most common presentation. Seven patients had solitary lesion and 13 patients had multiple lesions. Eleven patients with multiple metastases were treated with palliative chemotherapy and radiotherapy. Patients who had solitary metastases to liver had a median survival time of 49 months versus 13.5 months for other patients $(p=0.001)$. Conclusion: Patients who presented with solitary liver metastases could be treated with a course of neoadjuvant chemo-radiotherapy similar to the curative one.
\end{abstract}

Keywords: Rectum; Chemo-radiation; Metastases

\section{Introduction}

Approximately $20 \%$ to $30 \%$ of patients with rectal cancer found to have metastatic lesions. Palliative treatment strategy for advanced stage rectal cancer should be individualized to patients according to performance status, stage and symptoms. Chemotherapy for metastatic disease is the standard therapy for asymptomatic patients. ${ }^{1-4}$ Symptomatic patients are considered to a challenge and can be treated with chemotherapy or combined chemo-radiation therapy in conjunction with a surgery, if necessary. The most common presentations of these patients were bleeding per rectum and bowel obstruction for which they need dedicated plan of palliative therapy which emphasize on symptoms relief and improving

Corresponding author: Mohamed Aboziada; Department of Radiation Oncology, South Egypt Cancer Institute, Assiut University, Egypt.

Cite this article as:

Aboziada MA, Attia AM, Alhamad AA. Presentation and outcome of twenty patients with synchronous stage IV rectal carcinoma. Int J Cancer Ther Oncol 2014; 2(3):020313.

DOI: $10.14319 / \mathrm{ijcto} .0203 .13$ quality of life. The choice of treatment is mainly dependent upon the patient's symptoms, age, co-morbid conditions, and extent of disease. A multidisciplinary approach which includes medical oncologist, radiation oncologist, palliative care and the surgeon of determining wither or not invasive intervention is indicated. ${ }^{5}$

The rationale for elective resection in asymptomatic patients is to prevent further complications. This could develop during the treatment course, which may require urgent surgery associated with higher morbidity and mortality. Recent agents of chemotherapy showed improvement in prospective to the survival among patients with stage IV colorectal cancer; however, the risks and benefits of a deferred surgical strategy have not been completely evaluated. Combinations of fluorouracil/leucovorin with oxaliplatin or irinotecan have yielded response rates of $50 \%$, disease control rates of $85 \%$, and a median survival rate of 20 months in prospective clinical trials. ${ }^{6,7}$ The purpose of this research is to review the presentation, outcome and prognostic factors for stage IV rectal patients at our institute. 


\section{Methods and Materials}

\section{Patient characteristics}

Our group consisted of 90 patients with confirmed histology of rectal carcinoma presented at our institute between January 2009 to December 2010. For all patients, medical charts, radiology films, radiotherapy charts and their follow up notes were reviewed after receiving the approval from Institutional Ethics Board. Of those patients, only 20 patients (22.2\%) had rectal carcinoma with distant metastases (DM). History and physical examination of each patient were obtained too. A full staging work up was done for all of the patients. Metastatic work up included CT chest, abdomen, and pelvis. Liver tri-phasic computed tomography (CT) and magnetic resonance imaging (MRI) of liver and bone scan were performed as necessary. Laboratory investigations included count blood cells, liver, and renal profiles. Tumor markers included carcino-embryonic antigen and CA 19-9.

\section{Chemo-Radiation therapy}

Patients underwent CT simulation in a prone position with distended bladder, belly board, and thermoplastic sheet fixation. Multiple CT cuts at $0.5 \mathrm{~cm}$ interval were obtained throughout the pelvis. CT data was transferred to the $\mathrm{XiO}$ treatment planning system (version 4.2). On each axial CT slice, clinical target volume (CTV) and organs at risk (OAR) were contoured. CTV included the rectum, mesorectum, internal iliac and pre-sacral lymph nodes. Planning target volume (PTV) was generated with a $0.5 \mathrm{~cm}$ expansion from the CTV to account for the setup errors. Appropriate field weighting and beam modifiers (wedges and blocks) were selected to keep the OAR doses below their tolerance. For beam arrangement in the treatment plans three-field technique (a posterior field and two opposed lateral fields) was used to spare the anterior structures, particularly the small bowel. All patients were treated by a high energy linear accelerator with photon energies 6 and/or $15 \mathrm{MV}$. The total dose was $50.4 \mathrm{~Gy} / 28$ fractions/ 5.5 weeks. Specifically, the primary plans had dose prescribed to the PTV with schema of $45 \mathrm{~Gy} / 25$ fractions in 5 weeks. The boost plans had dose of $5.4 \mathrm{~Gy}$ in 3 fractions was delivered to the gross tumor volume (GTV) plus $2 \mathrm{~cm}$ margin. Palliative radiotherapy to the pelvis (gross tumor plus 2-cm margin) was $30 \mathrm{~Gy} / 10$ fractions/2 weeks; however, palliative dose to bone was 20 $\mathrm{Gy} / 5$ fractions /one week. Opposed anterior/ posterior fields used for the palliative intent. Capcitabine was given concurrent with radical radiotherapy by a dose $825 \mathrm{mg} / \mathrm{m}^{2}$ twice daily. FOLFOX (fluorouracil, leucovorin, and oxaliplatin) regimen was given in the adjuvant setting for 4 months as well as the first line for multiple metastases till progression. The second line was FOLFIRI (fluorouracil, leucovorin, and irinotecan) regimen. The median number cycles of chemotherapy were 8 cycles for palliative intent. K-ras test was not available at our institute by that time; therefore, cetuximab was not used. Dose modification and treatment interruption were permissible.

\section{Statistical analysis}

Kaplan-Meier estimates of actuarial survival and progression free survival were calculated with Graphed prism program (version 5). For comparisons of survival differences, the log-rank test was performed. The $\mathrm{p}$-values were double-sided with $\mathrm{p} \leq 0.05$ considered statistically significant.

\section{Results}

TABLE 1: Patients' characteristics.

\begin{tabular}{llcc}
\hline \hline \multirow{2}{*}{ Sex } & & Number (20) & Percentage \\
& Male & 13 & 65 \\
& Female & 7 & 35 \\
\hline Tumor & T2 & 3 & 15 \\
& T3 & 8 & 40 \\
& T4 & 9 & 45 \\
\hline Node & N0 & 3 & 15 \\
& N1 & 14 & 70 \\
& N2 & 6 & 30 \\
\hline Well differentiated & 3 & 15 \\
Moderate differentiated & 5 & 25 \\
Poor differentiated & 3 & 15 \\
\hline Signet & 1 & 5 \\
Mucoid & 8 & 40 \\
\hline Site of metastases & & \\
Liver & 16 & 80 \\
Lung & 1 & 5 \\
Bone & 3 & 15 \\
\hline Presentation & & \\
Bleeding & 14 & 70 \\
Tenesmus & 4 & 20 \\
Pain & 10 & 50 \\
Obstruction & 8 & 40 \\
\hline \hline
\end{tabular}

All patients were less than 40 years of age. The patient group included 13 males and 7 females. Bleeding per rectum, pain, and bowel obstruction were the most common presenting symptoms (Table 1). Most of patients presented with multiple symptoms. Rectal biopsy revealed that out of 20 patients, 8 patients had mucoid carcinoma, 1 patient had signed ring carcinoma while adenocarcinoma was found in 11 patients. According to TNM staging system, $45 \%$ of cases had T4 tumors and $85 \%$ had lymph node metastasis at the time of diagnosis. Liver represents the most common site of distant metastases; 7 patients had solitary lesion and 13 patients had multiple lesions.

Patients who presented with solitary liver lesions treated with neo-adjuvant chemo-radiation (capcitabine + radiotherapy) followed by surgery including tumor resection + metastatectomy then followed by adjuvant chemotherapy. In regard of radical surgery, 3 patients underwent low anterior resection and 4 patients underwent abdomen-perineal resection (APR). Patients who presented with multiple metastases 
were treated with palliative intension. Eleven patients of them (11 out of 13) were treated with palliative chemotherapy and palliative radiotherapy (RT). Two patients treated with best supportive care due to poor performance status. Palliative APR was done for two out of these 13 patients. No patient needed late palliative surgery.

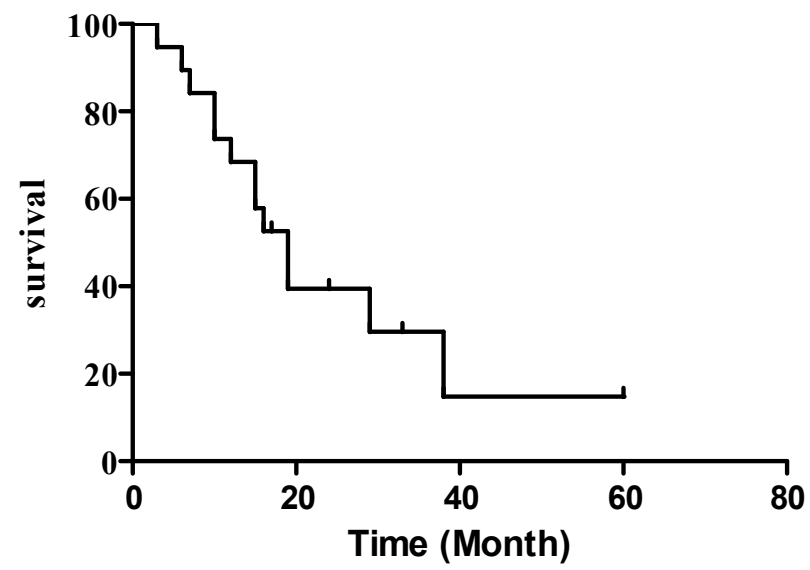

FIG.1: Overall Survival Proportion.

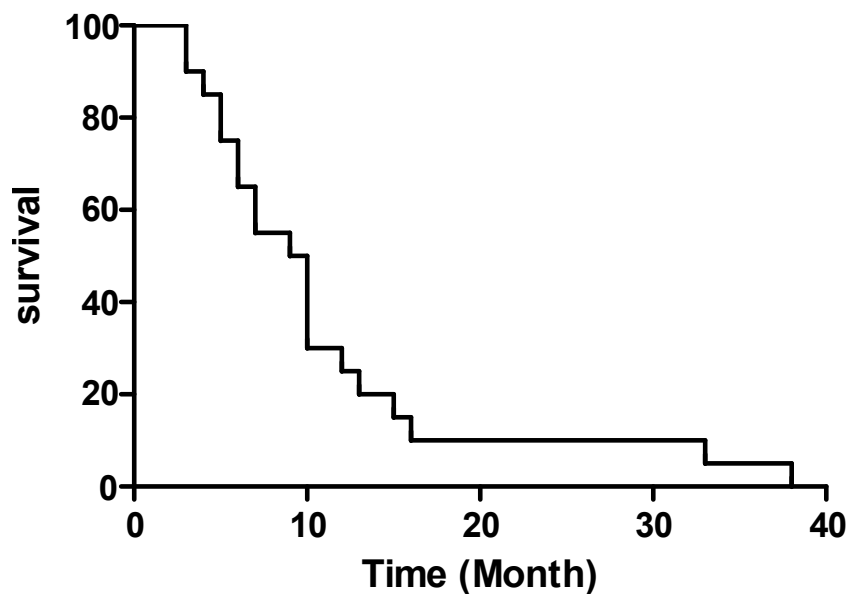

FIG. 2: Progression Free Survival.

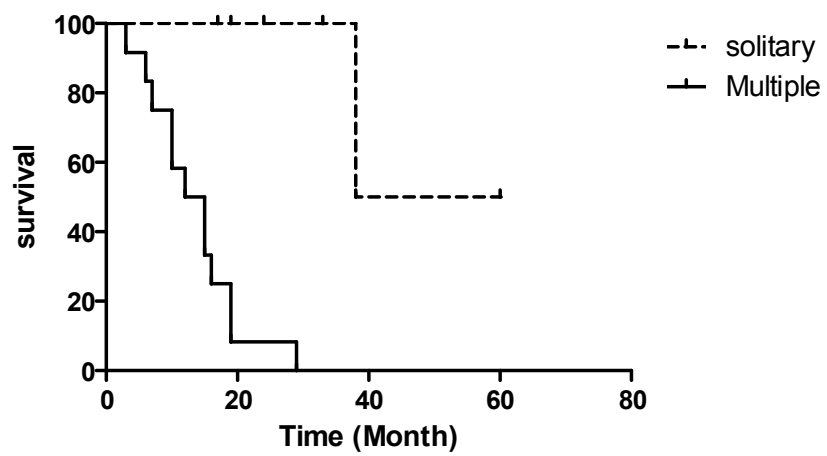

FIG. 3: Overall Survival of solitary and multiple metastases.

The median follow up period for all patients was 9.5 months (range 4-60). The median survival for the whole group of patients was 19 months (Figure 1). The median time of pro- gression free survival was 9.5 months for all patients (Figure 2). For patients who had solitary liver metastases, the median survival time was 49 months versus 13.5 months for patients who had multiple metastases $(\mathrm{p}=0.001)$ (Figure 3 ).

\section{Discussion}

The management of patients with metastatic colo-rectal carcinoma is still controversial, while for patients with solitary hepatic lesions the beneficiary of radical therapy has been shown. There was no benefit of any radical therapy for patients with diffuse metastatic spread. Rosen et al. ${ }^{8}$ favored an aggressive approach resection of the primary tumor in all patients in the absence of extensive metastases or carcinomatosis. More than two thirds of patients who presented with synchronous, stage IV colorectal cancer in the United States underwent up-front surgical resection of their primary tumors. ${ }^{9,10}$ Retrospective analyses in the pre-target therapy era have shown that resection of asymptomatic primary tumors was frequently associated with prolonged survival, but was not found to significantly reduce the incidence of life-threatening tumor-related complications. ${ }^{11-13}$ However, Poultsides et al ${ }^{14}$ demonstrated that 217 patients out of 233 patients (92\%) never required surgical palliation of their primary tumor when received upfront modern combination chemotherapy. Ten patients (4\%) required non-surgical intervention such as stent or RT for symptomatic management of the primary site. Neither use of bevacizumab, location of the primary tumor in the rectum, or metastatic disease burden was associated with increased intervention rate. Compared to our sample, only two patients underwent initial palliative resection.

Upfront surgery has historically been opted for these patients to prevent acute bowel obstruction, bleeding or perforation. As well known, an emergent surgery is usually associated with higher mortality. In our study late surgery was not required for any of the patients. The rate of late surgical intervention necessary to palliate primary tumor-related events is $7 \%$ to $20 \% .{ }^{10,12-14}$ Other reason for prophylactic surgery was that patients with low metastatic burden who are presumed to be at high risk of obstruction because of their relatively long survival times. ${ }^{8}$ Other reports, however, have failed to establish a correlation between advanced disease stage and the incidence of late primary tumor complications. ${ }^{13,15}$ Radiotherapy or chemo-radiotherapy can be given as palliative intent. Objective tumor regression can be produced and symptoms can be relieved. Radiotherapy is particularly effective for controlling pain, bleeding and discharge but often less effective for altered bowel habit. ${ }^{16,17}$

Patients underwent a palliative treatment have a relatively short survival duration (median: 6-9 months), with dismal 5-years survival rates (0\%-5\%). ${ }^{17}$ This is mainly for patients who present with symptoms include obstruction, pain, bleeding and perforation. New evolving regimen of systemic 
therapy have expanded the therapeutic options for these patients and improved median survival from less than one year to 20 months or longer. Fewer than $10 \%$ of those patients treated with chemotherapy alone are still alive at five years ${ }^{18-21}$, which is matched with our study. The median survival for patients with multiple metastases was 13.5 months. No survival advantage is gained by resection of an asymptomatic primary lesion in the setting of incurable stage IV colorectal cancer. The median survival was 14.5 months for patients who underwent tumor resection versus 16.6 months for patients who did not underwent any surgical resection. ${ }^{11,18,22,23}$

Liver is the dominant metastatic site for patients with colo-rectal cancer. Twenty percent of patients with isolated hepatic metastases are candidates to potentially curative resection. Still the majority of patients are not surgical candidates because of tumor size, location, multifocality, or inadequate hepatic reserve. Resection offers the greatest likelihood of cure for patients with isolated liver metastases. ${ }^{24,25}$ In our review, the group who presented with solitary liver metastases and had metastatectomy achieved a median survival 49 months. Five-year survival rates after resection range from $24 \%$ to $58 \%{ }^{26-28}$ Five-year survival rate was $25 \%$ in a population-based retrospective report of 3957 United State Medicare enrollees undergoing hepatic resection for colorectal cancer liver metastases. ${ }^{29}$

The use of 3-dimension conformal radiation therapy (3DCRT) has been shown to produce less favorable clinical and dosimetric results when compared to the intensity modulated radiation therapy (IMRT) and volumetric modulated arc therapy (VMAT). ${ }^{30}, 31$ The use of advanced treatment techniques such as IMRT and VMAT could improve the clinical results presented in our study. In radiation therapy planning, accuracy of dose calculation is also equally important in order to avoid miscalculation of the dose. Since dose calculation algorithms can affect the clinical and dosimetric results in radiation therapy, it is recommended to use the more accurate dose calculation engines while computing the cancer treatment plans. ${ }^{32,33}$

\section{Conclusion}

Upfront chemo-radiation may be effective for metastatic rectal cancer patients to prevent the need of urgent surgery. Aggressive surgery with chemo-radiation is the hope of cure for solitary liver metastatic rectal carcinoma.

\section{Conflict of interest}

The authors declare that they have no conflicts of interest. The authors alone are responsible for the content and writing of the paper.

\section{References}

1. Surveillance, Epidemiology and End Results Program (www.seer.cancer.gov) SEER* Stat Database: Incidence - SEER 17 Regs Limited-Use, Nov 2006 Sub (1973-2004 varying), National Cancer Institute, DCCPS, Surveillance Research Program, Cancer Statistics Branch, released April 2007, based on the November 2006 submission.

2. Edge, SB, Byrd, DR, Compton, CC, et al (Eds). AJCC (American Joint Committee on Cancer) Cancer Staging Manual, $7^{\text {th }}$ edition, New York: Springer; 2010, p.143.

3. Siegel R, Naishadham D, Jemal A. Cancer statistics, 2013. CA Cancer J Clin 2013; 63:11-30.

4. Rothenberger DA. Palliative therapy of rectal cancer. Overview: epidemiology, indications, goals, extent, and nature of work-up. J Gastrointest Surg 2004; 8: 259-61.

5. Ronnekleiv-Kelly SM, Kennedy GD. Management of stage IV rectal cancer: Palliative options. World J Gastroenterol 2011; 17: 835-47.

6. Tournigand C, Andre T, Achille E, et al. FOLFIRI followed by FOLFOX6 or the reverse sequence in advanced colorectal cancer: a randomized GERCOR study. J Clin Oncol 2004; 22:229-37.

7. Goldberg RM, Sargent DJ, Morton RF, et al. A randomized controlled trial of fluorouracil plus leucovorin, irinotecan, and oxaliplatin combinations in patients with previously untreated metastatic colorectal cancer. J Clin Oncol 2004; 22:23-30.

8. Rosen SA, Buell JF, Yoshida A, Kazsuba S, et al. Initial presentation with stage IV colorectal cancer: how aggressive should we be? Arch Surg 2000; 135:530-5.

9. Temple LK, Hsieh L, Wong WD, et al. Use of surgery among elderly patients with stage IV colorectal cancer. JClin Oncol 2004; 22:3475-84.

10. Cook AD, Single R, McCahill LE. Surgical resection of primary tumors in patients who present with stage IV colorectal cancer: An analysis of Surveillance, Epidemiology, and End Results data, 1988 to 2000. Ann Surg Oncol 2005; 12:637-45.

11. Eisenberger A, Whelan RL, Neugut AI. Survival and symptomatic benefit from palliative primary tumor resection in patients with metastatic colorectal cancer: a review. Int J Colorectal Dis 2008; 23:559-68.

12. Tebbutt NC, Norman AR, Cunningham D, Hill ME, et al. Intestinal complications after chemotherapy for patients with unresected primary colorectal cancer and synchronous metastases. Gut 2003; 52:568-73. 
13. Scoggins CR, Meszoely IM, Blanke CD, et al. Nonoperative management of primary colorectal cancer in patients with stage IV disease. Ann SurgOncol 1999; 6:651-7.

14. Poultsides GA, Servais EL, Saltz LB, et al. Outcome of primary tumor in patients with synchronous stage IV colorectal cancer receiving combination chemotherapy without surgery as initial treatment. JClin Oncol 2009; 27:3379-84.

15. Sarela AI, Guthrie JA, Seymour MT, et al. Non-operative management of the primary tumour in patients with incurable stage IV colorectal cancer. Br J Surg 2001; 88:1352-6.

16. Hatfield P, Sebag-Montefiore D. The use of radiotherapy in rectal cancer. Scand J Surg 2003; 92: 65-73.

17. Saltz LB. Palliative management of rectal cancer: The roles of chemotherapy and radiation therapy. $J$ Gastrointest Surg 2004; 8: 274-6.

18. Scheithauer W, Rosen H, Kornek GV, et al. Randomized comparison of combination chemotherapy plus supportive care with supportive care alone in patients with metastatic colorectal cancer. BMJ 1993; 306:752-5.

19. Simmonds PC. Palliative chemotherapy for advanced colorectal cancer: Systematic review and meta-analysis. Colorectal Cancer Collaborative Group. BMJ2000; 321:531-5.

20. Sanoff HK, Sargent DJ, Campbell ME, et al. Five-year data and prognostic factor analysis of oxaliplatin and irinotecan combinations for advanced colorectal cancer: N9741. J Clin Oncol 2008; 26:5721-7.

21. Renouf DJ, Lim HJ, Speers C, et al. Survival for metastatic colorectal cancer in the bevacizumab era: a population-based analysis. Clin Colorectal Cancer 2011; 10:97-101.

22. Stelzner S, Hellmich G, Koch R, Ludwig K. Factors predicting survival in stage IV colorectal carcinoma patients after palliative treatment: a multivariate analysis. J SurgOncol 2005; 89: 211-7.

23. Scoggins CR, Meszoely IM, Blanke CD, et al. Non operative Management of Primary Colorectal Cancer in Patients with Stage IV Disease. Ann Surg Oncol 1999; 6: 651-7.

24. Wei AC, Greig PD, Grant D, et al. Survival after hepatic resection for colorectal metastases: a 10-year experience. Ann Surg Oncol 2006; 13:668-76.

25. Simmonds PC, Primrose JN, Colquitt JL, et al. Surgical resection of hepatic metastases from colorectal cancer: a systematic review of published studies. Br J Cancer 2006; 94:982-99.

26. Rees M, Tekkis PP, Welsh FK, et al. Evaluation of long-term survival after hepatic resection for meta- static colorectal cancer: a multifactorial model of 929 patients. Ann Surg 2008; 247:125-35.

27. Morris EJ, Forman D, Thomas JD, et al. Surgical management and outcomes of colorectal cancer liver metastases. Br J Surg 2010; 97:1110-8.

28. Robertson DJ, Stukel TA, Gottlieb DJ, et al. Survival after hepatic resection of colorectal cancer metastases: a national experience. Cancer 2009; 115:752-9.

29. Cummings LC, Payes JD, Cooper GS. Survival after hepatic resection in metastatic colorectal cancer: a population-based study. Cancer 2007; 109:718-26.

30. Arbea L, Ramos LI, Martínez-Monge R, et al. Intensity-modulated radiation therapy (IMRT) vs. 3D conformal radiotherapy (3DCRT) in locally advanced rectal cancer (LARC): dosimetric comparison and clinical implications. Radiat Oncol 2010; 5:17.

31. Teoh $\mathrm{M}$, Clark $\mathrm{CH}$, Wood $\mathrm{K}$, et al. Volumetric modulated arc therapy: a review of current literature and clinical use in practice. Br J Radiol 2011; 84:967-96.

32. Rana S. Clinical dosimetric impact of Acuros XB and analytical anisotropic algorithm (AAA) on real lung cancer treatment plans: review. Int J Cancer Ther Oncol 2014; 2:02019.

33. Lu L. Dose calculation algorithms in external beam photon radiation therapy. Int J Cancer Ther Oncol 2013; 1:01025. 